\title{
Symbolic integration with respect to the Haar measure on the unitary groups
}

\author{
Z. PUCHAŁA* and J.A. MISZCZAK \\ Institute of Theoretical and Applied Informatics, Polish Academy of Sciences, 5 Bałtycka Str., 44-100 Gliwice, Poland
}

\begin{abstract}
We present IntU package for Mathematica computer algebra system. The presented package performs a symbolic integration of polynomial functions over the unitary group with respect to unique normalized Haar measure. We describe a number of special cases which can be used to optimize the calculation speed for some classes of integrals. We also provide some examples of usage of the presented package.
\end{abstract}

Key words: unitary group, Haar measure, circular unitary ensemble, symbolic integration.

\section{Introduction}

The integration over unitary group is an important subject of studies in many areas of science, including mathematical physics, random matrix theory and algebraic combinatorics. In 2006 Collins and Śniady [1] proved a formula for calculating monomial integrals with respect to the Haar measure on the unitary group

$\int_{\mathrm{U}(d)} U_{I J} \bar{U}_{I^{\prime} J^{\prime}} d U=\int_{\mathrm{U}(d)} U_{i_{1} j_{1}} \ldots U_{i_{n} j_{n}} \bar{U}_{i_{1}^{\prime} j_{1}^{\prime}} \ldots \bar{U}_{i_{n}^{\prime} j_{n}^{\prime}} d U$

Integrals of the above type, called moments of the $\mathrm{U}(d)$, have been known in mathematical physics literature for a long time. The problem of the integration of elements of unitary matrices was considered for the first time in the context of nuclear physics in [2]. The asymptotic behaviour of the integrals of the type (1) was considered by Weingarten in [3].

In this paper we describe IntU, a Mathematica package [4] for calculating polynomial integrals over $\mathrm{U}(d)$ with respect to the Haar measure. We describe a number of special cases which can be used to optimize the calculation speed for some classes of integrals. We also provide some examples of usage of the presented package, including the applications in the study of the geometry of the quantum states.

This paper is organised as follows. In Section 2, we introduce notation and present the mathematical background of polynomial integrals over unitary group. In Section 3, we describe some special cases, in which the integration can be calculated more efficiently. In Section 4 we provide the description of IntU package with the list of main functions. In Section 5 we show some examples of the usage. In Section 6 we provide a summary of the presented results and conclusions.

*e-mail: z.puchala@iitis.pl

\section{Mathematical background}

2.1. Basic concepts. By $M_{n}$ we denote square matrices of size $n$. The compact group of $d \times d$ unitary matrices is denoted as $\mathrm{U}(d)$. We equip the above group with unique normalized Haar measure denoted by $d U$. Random elements distributed with measure $d U$ form the so-called circular unitary ensemble.

Integer partition $\lambda$ of a positive integer $n$ is a weakly decreasing sequence $\lambda=\left(\lambda_{1}, \lambda_{2}, \ldots, \lambda_{l}\right)$ of positive integers, such that $\sum_{i=1}^{l} \lambda_{i}=|\lambda|=n$. To denote that $\lambda$ is a partition of $n$, we write $\lambda \vdash n$. The length of a partition is denoted by $l(\lambda)$. By $\lambda \sqcup \mu$ we denote a partition of $n_{1}+n_{2}$ obtained by joining partitions $\lambda \vdash n_{1}$ and $\mu \vdash n_{2}$.

Each permutation $\sigma \in S_{n}$ can be uniquely decomposed into a sum of disjoint cycles, where the lengths of the cycles sum up to $n$. Thus the vector of the lengths of the cycles, after reordering, forms a partition $\lambda \vdash n$. The partition $\lambda$ is called the cycle type of $\sigma$ permutation.

2.2. Moments of the $\mathbf{U}(\boldsymbol{d})$. Let us consider a polynomial $p$. From the linearity of an integral we have

$$
\int_{\mathrm{U}(d)} p(U) d U=\sum_{I, J, I^{\prime}, J^{\prime}} c\left(I, J, I^{\prime}, J^{\prime}\right) \int_{\mathrm{U}(d)} U_{I J} \bar{U}_{I^{\prime} J^{\prime}} d U
$$

where $I, J, I^{\prime}, J^{\prime}$ are multi-indices and $c$ are the coefficients of $p$. The value of such monomial integrals is given as [1]

$$
\begin{aligned}
& \int_{\mathrm{U}(d)} U_{I J} \bar{U}_{I^{\prime} J^{\prime}} d U=\int_{\mathrm{U}(d)} U_{i_{1} j_{1}} \ldots U_{i_{n} j_{n}} \bar{U}_{i_{1}^{\prime} j_{1}^{\prime}} \ldots \bar{U}_{i_{n}^{\prime} j_{n}^{\prime}} d U= \\
& =\sum_{\sigma, \tau \in S_{n}} \delta_{i_{1}, i_{\sigma(1)}^{\prime}} \ldots \delta_{i_{n}, i_{\sigma(n)}^{\prime}} \delta_{j_{1}, j_{\tau(1)}^{\prime}} \ldots \delta_{j_{n}, j_{\tau(n)}^{\prime}} W g\left(\tau \sigma^{-1}, d\right),
\end{aligned}
$$

where $W g$ is the Weingarten function discussed below. In the case of the multi-indices differing in length, i.e. $n \neq n^{\prime}$, we have 


$$
\begin{aligned}
& \int_{\mathrm{U}(d)} U_{I J} \bar{U}_{I^{\prime} J^{\prime}} d U= \\
& =\int_{\mathrm{U}(d)} U_{i_{1} j_{1}} \ldots U_{i_{n} j_{n}} \bar{U}_{i_{1}^{\prime} j_{1}^{\prime}} \ldots \bar{U}_{i_{n^{\prime}}^{\prime} j_{n^{\prime}}^{\prime}} d U=0 .
\end{aligned}
$$

The integrals of the above type are known as moments of the $\mathrm{U}(d)$.

2.3. Weingarten function. The Weingarten function [1] is defined for $\sigma \in S_{n}$ and positive integer $d$ as

$$
W g(\sigma, d)=\frac{1}{(n !)^{2}} \sum_{\substack{\lambda \vdash n \\ l(\lambda) \leq d}} \frac{\chi^{\lambda}(e)^{2}}{s_{\lambda, d}(1)} \chi^{\lambda}(\sigma),
$$

where the sum is taken over all integer partitions of $n$ with length $l(\lambda) \leq d, s_{\lambda, d}(1)$ is the Schur polynomial $s_{\lambda}$ evaluated at $(\underbrace{1,1, \ldots, 1}_{d})$ and $\chi^{\lambda}$ is an irreducible character of the symmetric group $S_{n}$ indexed by partition $\lambda$.

2.3.1. The dimension of irreducible representation of $U(d)$. The value of the Schur polynomial at the point $(\underbrace{1,1, \ldots, 1}_{d})$, i.e. the dimension of irreducible representation of $\mathrm{U}(d)$ corresponding to partition $\lambda$, is equal to e.g. Theorem 6.3 in [5]

$$
s_{\lambda, d}(1)=s_{\lambda}(\underbrace{1,1, \ldots, 1}_{d})=\prod_{1 \leq i<j \leq d} \frac{\lambda_{i}-\lambda_{j}+j-i}{j-i} .
$$

2.3.2. Irreducible character of $\boldsymbol{S}_{\boldsymbol{n}}$. The irreducible character of $S_{n}$ indexed by partition $\lambda, \chi^{\lambda}(\sigma)$ depends on a conjugacy class of permutation $\sigma$. Two permutations are in the same conjugacy class if and only if they have the same cycle type, thus it is common to write $\chi^{\lambda}(\sigma)=\chi^{\lambda}(\mu)$, where $\mu$ is an integer partition corresponding to the cycle type of $\sigma$.

In the case of identity permutation the cycle type is given by a trivial partition, $e=(\underbrace{1,1, \ldots, 1}_{n})$, and the character value is equal to the dimension of the irreducible representation of $S_{n}$ indexed by $\lambda$. In this case it is given by the celebrated hook length formula (see Eq 4.12 in [5])

$$
\chi^{\lambda}(e)=\frac{|\lambda| !}{\prod_{i, j} h_{i, j}^{\lambda}},
$$

where $|\lambda|=\lambda_{1}+\lambda_{2}+\cdots+\lambda_{l(\lambda)}$, and $h_{i, j}^{\lambda}$ is the hook length of the cell $(i, j)$ in a Ferrers diagram corresponding to partition $\lambda[6]$.

In the case of a non-trivial partition the character of symmetric group $\chi^{\lambda}(\sigma)=\chi^{\lambda}(\mu)$ can be evaluated with the use of Murnaghan-Nakayama rule (see Theorem. 4.10.2 in [7]), which describes a combinatorial way of calculating the character; [8] is used.
From the above considerations one can deduce that the Weingarten function depends only on a cycle type of a permutation $\sigma$ and thus it is constant on a conjugacy class represented by $\sigma$. Thus we may define the Weingarten function as

$$
W g(\mu, d)=\frac{1}{(|\mu| !)^{2}} \sum_{\substack{\lambda \vdash|\mu| \\ l(\lambda) \leq d}} \frac{\chi^{\lambda}(e)^{2}}{s_{\lambda, d}(1)} \chi^{\lambda}(\mu),
$$

where $\mu$ is an integer partition, which is a cycle type of $\sigma$.

\section{Special cases}

In this section we present some special cases of integrals with respect to the Haar measure on the unitary group. In these cases the value of the integral can be calculated without the direct usage of (3), which requires processing of $\prod_{i}^{d} k_{i} ! \prod_{j}^{d} l_{j}$ ! permutations, where $k_{i}\left(l_{j}\right)$ denotes the number of $i(j)$ in multi-indices $I(J)$, respectively.

The presented special cases have been implemented in the package to increase its efficiency. This goal has been achieved by minimizing the number of calculations of the Weingarten function.

3.1. First two moments of $\mathbf{U}(\boldsymbol{d})$. In the case of monomials of rank equal to 2 and 4, from [9] we have

$$
\int_{\mathrm{U}(d)} u_{i j} \bar{u}_{i^{\prime} j^{\prime}} d U=\frac{1}{d} \delta_{i i^{\prime}} \delta_{j j^{\prime}}
$$

and

$$
\begin{aligned}
\int_{\mathrm{U}(d)} & u_{i_{1} j_{1}} u_{i_{2} j_{2}} \bar{u}_{i_{1}^{\prime} j_{1}^{\prime}} \bar{u}_{i_{2}^{\prime} j_{2}^{\prime}} d U= \\
= & \frac{\delta_{i_{1} i_{1}^{\prime}} \delta_{i_{2} i_{2}^{\prime}} \delta_{j_{1} j_{1}^{\prime}} \delta_{j_{2} j_{2}^{\prime}}+\delta_{i_{1} i_{2}^{\prime}} \delta_{i_{2} i_{1}^{\prime}} \delta_{j_{1} j_{2}^{\prime}} \delta_{j_{2} j_{1}^{\prime}}}{d^{2}-1}+ \\
- & \frac{\delta_{i_{1} i_{1}^{\prime}} \delta_{i_{2} i_{2}^{\prime}} \delta_{j_{1} j_{2}^{\prime}} \delta_{j_{2} j_{1}^{\prime}}+\delta_{i_{1} i_{2}^{\prime}} \delta_{i_{2} i_{1}^{\prime}} \delta_{j_{1} j_{1}^{\prime}} \delta_{j_{2} j_{2}^{\prime}}}{d\left(d^{2}-1\right)}
\end{aligned}
$$

allowing calculation of the values for polynomial integrals of degree less than 5 without the direct usage of (3).

This optimization gives only a minor improvement of efficiency, as in these cases the direct calculation of Weingarten function is very fast.

3.2. Elements from one row (column). The next optimization is based on the fact that the distribution of random vector consisting of squares of absolute values of a row (or a column) of unitary matrix distributed with the Haar measure, is uniform on a standard $d$-simplex $\Delta^{d}[9]$

$$
\left\{\left|u_{i, 1}\right|^{2},\left|u_{i, 2}\right|^{2}, \ldots,\left|u_{i, d}\right|^{2}\right\} \sim \mathbb{U}\left(\Delta^{d}\right),
$$

where $\mathbb{U}(A)$ denotes the normalized uniform measure (proportional to Lebesgue measure) on a set $A \subset \mathbb{R}^{d}$, and standard $d$-simplex $\Delta^{d}$ is defined as, $\Delta^{d}=\left\{\lambda \in \mathbb{R}^{d}: \lambda_{i} \geq 0, \sum_{i=1}^{d} \lambda_{i}=1\right\}$. 
Using Beta integral, one obtains that for a fixed row $i_{0}$ and a vector $p$ with non-negative entries $p_{j}$, we have the following

$$
\begin{aligned}
& \int_{\mathrm{U}(d)} \prod_{j=1}^{d}\left|u_{i_{0}, j}\right|^{2 p_{j}} d U= \\
& =\Gamma(d) \frac{\Gamma\left(p_{1}+1\right) \times \cdots \times \Gamma\left(p_{d}+1\right)}{\Gamma\left(p_{1}+\cdots+p_{d}+d\right)} .
\end{aligned}
$$

The above, as a special case, gives:

$$
\begin{aligned}
\int_{\mathrm{U}(d)}\left|u_{i_{0}, j}\right|^{2 k} d U & =\frac{(d-1) ! k !}{(d-1+k) !}, \\
\int_{\mathrm{U}(d)}\left|u_{i_{0}, j}\right|^{2}\left|u_{i_{0}, k}\right|^{2} d U & =\frac{1}{d(d+1)},
\end{aligned}
$$

which can be found in literature $[9,10]$.

This optimization allows for an enormous improvement in efficiency thanks to avoiding $\left(\sum p_{i}\right) ! \prod p_{i}$ ! executions of Weingarten function needed in the case of the direct usage of formula (3).

3.3. Even powers of diagonal element absolute values. In this subsection we consider the integrals of the type

$$
\int_{\mathrm{U}(d)}\left|u_{i, j}\right|^{2 p}\left|u_{k, l}\right|^{2 q} d U
$$

where $p, q$ are non-negative integers and $i \neq k, j \neq l$. In the case of $p=q=1$ this integral is known [9]

$$
\int_{\mathrm{U}(d)}\left|u_{i, j}\right|^{2}\left|u_{k, l}\right|^{2} d U=\frac{1}{d^{2}-1} .
$$

For general non-negative integers $p, q$ the following proposition is true.

Proposition 1. Let $p, q$ be non-negative integers, for $i \neq k$ and $j \neq l$, we have

$$
\int_{\mathrm{U}(d)}\left|u_{i, j}\right|^{2 p}\left|u_{k, l}\right|^{2 q} d U=p ! q ! \sum_{\substack{\lambda \vdash p \\ \mu \vdash q}} \mathbf{k}_{\lambda} \mathbf{k}_{\mu} W g(\lambda \sqcup \mu, d),
$$

where the above sum is taken over all integer partitions of $p$ and $q$. Symbol $\mathbf{k}_{v}$ denotes a cardinality of conjugacy class for a permutation with cycle type given by partition $v \vdash r$ [7]

$$
\mathbf{k}_{v}=\frac{r !}{1^{m_{1}} m_{1} ! 2^{m_{2}} m_{2} ! \cdots r^{m_{r}} m_{r} !}
$$

where $m_{i}$ denotes the number of $i$ in partition $v$.

The above is a special case of a general fact.
Proposition 2. For any permutation $\pi \in S_{d}$ and any non-negative integers $p_{1}, p_{2}, \ldots, p_{d}$, we have

$$
\begin{gathered}
\int_{\mathrm{U}(d)} \prod_{j=1}^{d}\left|u_{j, \pi(j)}\right|^{2 p_{j}} d U=\int_{\mathrm{U}(d)} \prod_{j=1}^{d}\left|u_{j, j}\right|^{2 p_{j}} d U= \\
=\left(\prod_{j=1}^{d} p_{j} !\right) \sum_{\lambda_{1} \vdash p_{1}} \sum_{\lambda_{2} \vdash p_{2}} \ldots \sum_{\lambda_{d} \vdash p_{d}}\left(\prod_{j=1}^{d} \mathbf{k}_{\lambda_{j}}\right) \times \\
\times W g\left(\lambda_{1} \sqcup \lambda_{2} \sqcup \cdots \sqcup \lambda_{d}, d\right) .
\end{gathered}
$$

Proof. If we apply the formula from Eq. (3) to integral (19), then the non-vanishing permutations of indices are those which permute within the blocks of sizes $\left\{p_{1}, p_{2}, \ldots, p_{d}\right\}$, i.e.

$$
\sigma=\sigma_{1} \oplus \sigma_{2} \oplus \sigma_{d}
$$

where $\sigma_{j} \in S_{p_{j}}$, permuting indices in $j^{\text {th }}$ block of size $p_{j}$. The same situation holds in the case of the second indices. Thus the permutation $\tau \sigma^{-1}$ is also in this form. Each permutation of the above type will be present in the sum $\prod_{j=1}^{d} p_{j}$ ! times. The cycle type of such permutations is given by a partition which is obtained by joining cycle types of small permutations. Since Weingarten function depends only on a cycle type of permutation, the size of conjugacy class is calculated for each partition, instead of evaluating Weingarten function multiple times.

The formula (19) is far less computationally demanding than the direct usage of (3). One can notice that Proposition 2 allows us to avoid at least $\prod_{j=1}^{d} p_{j}$ ! executions of Weingarten function comparing to the direct usage of (3). However, the exact time-efficiency depends also on the cardinality of conjugacy classes for partitions of $p_{1}, \ldots, p_{d}$.

3.4. Cycle permutations. In this section we consider another special type of integral for positive integer $k$ and a permutation $\sigma$ of $\{1,2, \ldots, m\}$ being a cycle

$$
\int_{\mathrm{U}(d)}\left(\prod_{i=1}^{m} u_{i, i} \bar{u}_{i, \sigma(i)}\right)^{k} d U
$$

We have the following proposition.

Proposition 3. Let $m \leq d, k$ is a positive integer and $\sigma \in S_{m}$ be a cycle, i.e. the cycle type is given by partition $\{m\}$. Then

$$
\begin{aligned}
& \int_{\mathrm{U}(d)}\left(\prod_{i=1}^{m} u_{i, i} \bar{u}_{i, \sigma(i)}\right)^{k} d U= \\
& =(k !)^{2 m-1} \sum_{\lambda \vdash k} \mathbf{k}_{\lambda} W g(m \lambda, d) .
\end{aligned}
$$

Proof. One can notice that cycle lengths of permutations in this setting must be divisible by $m$. The number of permutations 
with cycle type given by a particular partition can be easily obtained by the usual counting argument.

Using (21) one obtains an enormous improvement in efficiency by avoiding more than $(k !)^{2 m-1}$ executions of Weingarten function as compared to the case of the direct usage of (3).

\section{Package description}

Below we describe the functions implemented in IntU package. The functions are grouped in three categories: functions implementing the main functionality, functions related to the calculation of Weingarten function and helper functions.

4.1. Main functionality. The main functionality of $\operatorname{Int} U$ package is provided be the IntegrateUnitaryHaar and IntegrateUnitaryHaarIndices functions. The first one operates directly on polynomial expressions, while the second one accepts four-tuple of indices. The examples of the usage are given in Section 5.

- IntegrateUnitaryHaar [integrand, \{var,dim\}] - gives the definite integral on unitary group with respect to the Haar measure, accepting the following arguments

- integrand - the polynomial type expression of variable var with indices placed as subscripts, can contain any other symbolic expression of other variables,

- var - the symbol of variable for integration,

- dim - the dimension of a unitary group, must be a positive integer.

This function is presented in the examples described in Sections 5.1, 5.3 and 5.5.

- IntegrateUnitaryHaar $[f,\{u, d 1\},\{v, d 2\}, \ldots]$ - gives multiple integral

$$
\int_{\mathrm{U}(\mathrm{d} 1)} d U \int_{\mathrm{U}(\mathrm{d} 2)} d V \ldots f
$$

This function is presented in the example described in Section 5.4 .

- IntegrateUnitaryHaarIndices [ $\{\mathrm{I} 1, \mathrm{~J} 1, \mathrm{I} 2, \mathrm{~J} 2\}$, dim ] - calculates the integral in (3) for given multi-indices I1, J1, I2, J2 and the dimension dim of the unitary group. This function is presented in the example described in Section 5.2 .

4.2. Weingarten function. The main functions implemented in the package, IntegrateUnitaryHaar and IntegrateUnitaryHaarIndices, utilize the following functions to find the value of the integral.

- Weingarten [type, dim] - returns the value of the Weingarten function given in Eq. (8) and accepts the following arguments

- type - an integer partition which corresponds to cycle type of permutation (see Section 2.3)

- dim - the dimension of a unitary group, which must be a positive integer.
- CharacterSymmetricGroup [part, type] - gives the character of the symmetric group $\chi^{\text {part }}$ (type) (see Section 2.3.2).

Parameter type is optional. The default value is set to a trivial partition and in this case the function returns the dimension of the irreducible representation of symmetric group indexed by part, given by (7). If type is specified, the value of the character is calculated by Murnaghan-Nakayama rule using MNInner algorithm provided in [8].

- SchurPolynomialAt 1 [part, dim] - returns the value of the Schur polynomial $s_{\text {part }}$ at point $(\underbrace{1,1, \ldots, 1}_{\text {dim }})$, i.e. the dimension of irreducible representation of $U(\mathrm{dim})$ corresponding to part, see (6).

\subsection{Helper functions.}

- PermutationTypePartition[perm] - gives the partition which represents the cycle type of the permutation perm (see Section 2.1).

- MultinomialBeta [p] - for a given $d$-dimensional vector of non-negative numbers $p_{1}, p_{2}, \ldots, p_{d}$ returns the value of multinomial Beta function defined as

$$
B(p)=\frac{\prod_{i=1}^{d} \Gamma\left(p_{i}\right)}{\Gamma\left(\sum_{i=1}^{d} p_{i}\right)} .
$$

This function is used in the optimization described in Section 3.2 .

- ConjugatePartition [part] - gives a conjugate of a partition part (see [5]). This function is used for calculating hook-length formula given by (7).

- CardinalityConjugacyClassPartition [part] - gives a cardinality of a conjugacy class for the permutation with cycle type given by partition part (see [7, Eq. 1.2]). This function is used in the optimization described in Section 3.3.

- BinaryPartition [part] - gives a binary representation of a partition part. This function is needed for the implementation of MNInner algorithm.

\section{Examples of use}

In order to present the main features of the described package, we provide a series of examples.

5.1. Elementary integrals. Let us assume that $d=3$. We want to calculate the following integrals

$$
\begin{aligned}
& \int_{\mathrm{U}(d)}\left|u_{1,1}\right|^{2} d U, \\
& \int_{\mathrm{U}(d)}\left|u_{1,1}\right|^{2}\left|u_{2,2}\right|^{2} d U, \\
& \int_{\mathrm{U}(d)} u_{1,1} u_{2,2} \bar{u}_{1,2} \bar{u}_{2,1} d U .
\end{aligned}
$$


Let us start by initializing the package

$\ln [1]:=<\operatorname{Int} \mathbf{U}^{-}$

Next, we calculate the integrals.

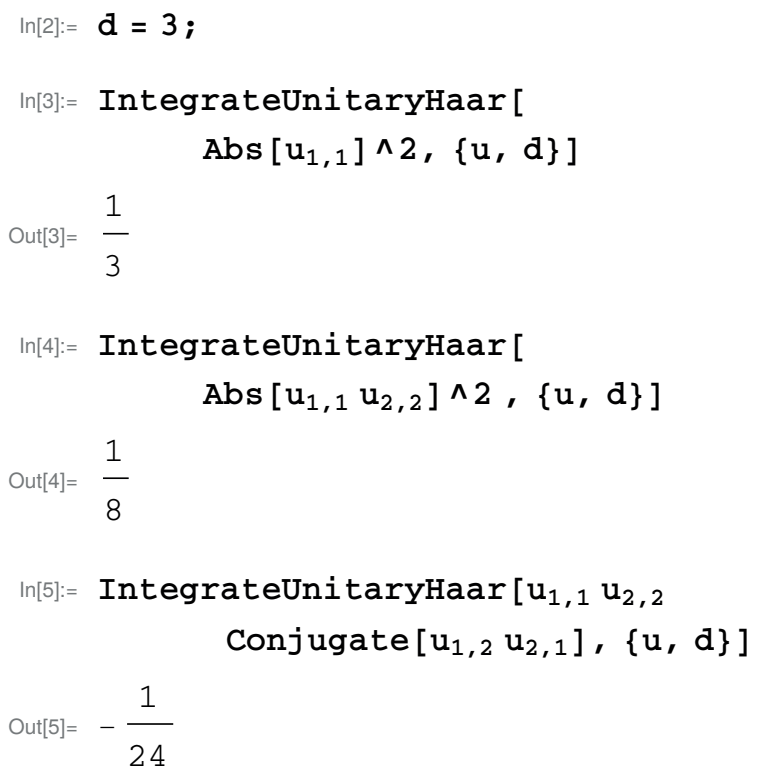

5.2. Operations on indices. Let us take the following set of multi-indices

$$
\begin{aligned}
I & =\{1,1,1,2,2\}, & J & =\{2,2,1,1,1\} \\
I^{\prime} & =\{1,1,1,2,2\}, & J^{\prime} & =\{2,1,1,2,1\}
\end{aligned}
$$

and set $d=6$. The above is equivalent to expression

$$
u_{1,2} u_{1,2} u_{1,1} u_{2,1} u_{2,1} \bar{u}_{1,2} \bar{u}_{1,1} \bar{u}_{1,1} \bar{u}_{2,2} \bar{u}_{2,1}
$$

with symbolic variable $u$, which we aim to integrate over $\mathrm{U}(d)$. After simplification the expression is equal to

$$
\left|u_{1,1}\right|^{2}\left|u_{1,2}\right|^{2}\left|u_{2,1}\right|^{2} u_{1,2} u_{2,1} \bar{u}_{1,1} \bar{u}_{2,2} \text {. }
$$
dices

After initializing the package and defining appropriate in-

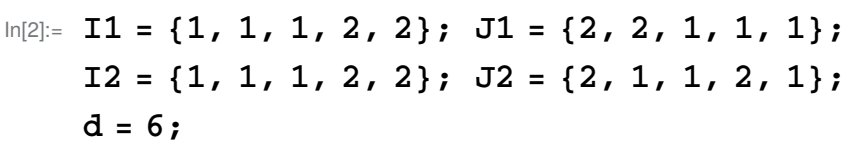

we calculate the integral using provided function IntegrateUnitaryHaarIndices as

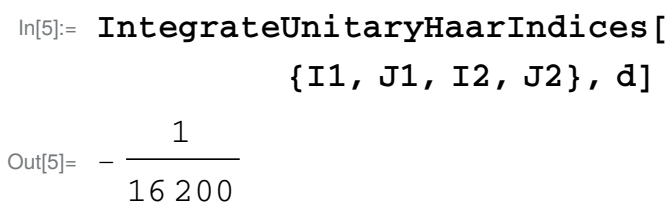

which is equivalent to

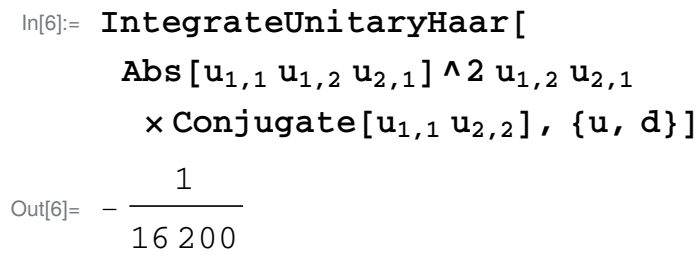

5.3. Matrix expressions. IntU package allows us to integrate matrix expressions, for example let us take $d=2$ and integrate

$$
\int_{\mathrm{U}(d)} U^{\otimes 2} \otimes \bar{U}^{\otimes 2} d U
$$

We define symbolic matrices $U \in \mathrm{U}(d)$ and $U 2=U \oplus U \in$ $\in \mathrm{U}\left(d^{2}\right)$ as

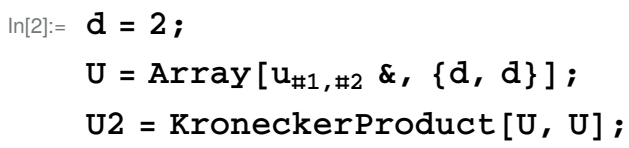

and construct the integrand as

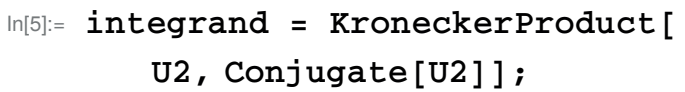

By using \{IntegrateUnitaryHaar\} function

$\ln [6]:=$ IntegrateUnitaryHaar [integrand, $\{\mathrm{u}, \mathrm{d}\}$ ]

we learn that the integral in (31) is equal to

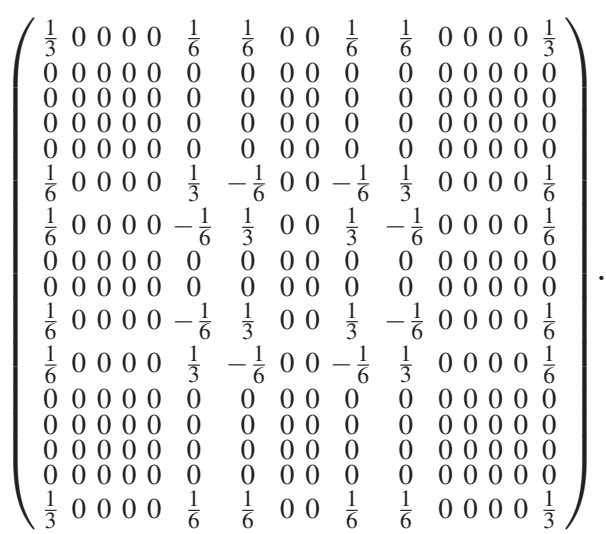

5.4. Mean value of local unitary orbit. In this example we calculate the mean value of a local unitary orbit of a given matrix $X \in M_{d^{2}}$

$$
\mathbb{E}\left[(U \otimes V) X(U \otimes V)^{\dagger}\right]
$$


We assume that $U$ and $V$ are stochastically independent random unitary matrices of size $d$ distributed with the Haar measure. In this case we have

$$
\begin{aligned}
& \mathbb{E}\left[(U \otimes V) X(U \otimes V)^{\dagger}\right]= \\
& =\int_{\mathrm{U}(d)} \int_{\mathrm{U}(d)}(U \otimes V) X(U \otimes V)^{\dagger} d U d V .
\end{aligned}
$$

In this example we take $d=3$.

We define symbolic matrices $X$ of size $d^{2}$ and $U, V \in \mathrm{U}(d)$ as

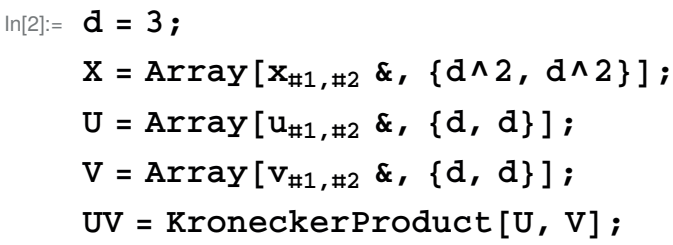

Using IntegrateUnitaryHaar function with two variable specifications, we calculate the double integral

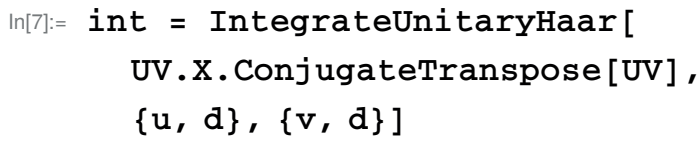

and find out that the expectation value in (33) is equal to

$$
\mathbb{E}\left[(U \otimes V) X(U \otimes V)^{\dagger}\right]=\frac{1}{d^{2}} \operatorname{tr} X \mathbb{1}_{d \times d}
$$

Similarly one can calculate the covariance tensor of the local unitary orbit of a given matrix $X \in M_{d^{2}}$. If $z=(U \oplus V)$ $X(U \oplus V)^{\dagger}$ then the covariance tensor is given by [11]

$$
\begin{aligned}
& \mathbb{E}\left[\left\{z_{i j} \bar{z}_{k l}\right\}_{i j k l}\right]= \\
& =\mathbb{E}\left[(U \otimes V) X(U \otimes V)^{\dagger} \otimes \overline{(U \otimes V) X(U \otimes V)^{\dagger}}\right] .
\end{aligned}
$$

Using IntegrateUnitaryHaar one can check that for the fixed dimension, the integral agrees with the calculations presented in [11].

5.5. Moments of maximally entangled numerical shadow. If $U$ is a random $d \times d$ unitary matrix distributed according to the Haar measure [12], then the pure state obtained by vectorization $|\xi\rangle=\frac{1}{\sqrt{d}} \operatorname{vec}(U)$ is maximally entangled on $H_{A} \oplus H_{B}=\mathbb{C}^{d} \oplus \mathbb{C}^{d}$. Moreover, state $|\xi\rangle$ has a distribution invariant to multiplication by local unitary matrices. We denote the corresponding probability measure on pure states of size $d \times d$ as $\mu$. The numerical shadow of operator $X \in M_{d^{2}}$ with respect to this measure (maximally entangled numerical shadow) is defined as

$$
P_{X}(z)=\int_{\mathbb{C}^{d^{2}}} \delta(z-\langle\psi|X| \psi\rangle) \mathrm{d} \mu(\psi)
$$

The corresponding probability measure is denoted by $d \mu_{X}^{E}$. For definition and basic facts concerning numerical shadows see $[11,13,14]$.

The first two moments of $d \mu_{X}^{E}$ are calculated in [11], and are given by

$$
\int_{\mathbb{C}} z d \mu_{X}^{E}(z)=\frac{1}{d^{2}} \operatorname{tr} X,
$$

and

$$
\begin{aligned}
& \int_{\mathbb{C}} z \bar{z} d \mu_{X}^{E}(z)=\frac{1}{d^{2}\left(d^{2}-1\right)}\left(|\operatorname{tr} X|^{2}+\|X\|_{\mathrm{HS}}^{2}\right)- \\
& -\frac{1}{d^{3}\left(d^{2}-1\right)}\left(\left\|\operatorname{tr}_{A}(X)\right\|_{\mathrm{HS}}^{2}+\left\|\operatorname{tr}_{B}(X)\right\|_{\mathrm{HS}}^{2}\right),
\end{aligned}
$$

where $\operatorname{tr}_{A}$ and $\operatorname{tr}_{B}$ denote partial traces over a specified sub-system and $\|\cdot\|_{H S}$ is a Hilbert-Schmidt norm given by $\|X\|_{\mathrm{HS}}=\sqrt{\operatorname{tr} X X^{\dagger}}$.

In order to calculate (39) and (39) define symbolic matrices

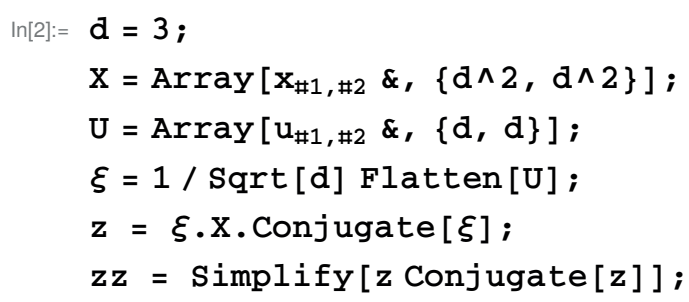

Now we calculate the first moment

$\operatorname{In}[8]:=$ IntegrateUnitaryHaar $[\mathbf{z},\{\mathrm{u}, \mathrm{d}\}]$

and the second moment

$\ln [9]:=$ int $=$ IntegrateUnitaryHaar $[\mathbf{z z},\{u, d\}]$

After some algebraic manipulations one can see that the above agrees with Eqs. (38) and (39).

IntU package has been applied successfully in the context of quantum entanglement in [15], where it was used to calculate the moments of the three-tangle.

\section{Summary}

We described IntU package for Mathematica computing system for calculating polynomial integrals over $\mathrm{U}(d)$ with respect to Haar measure. We described a number of special cases which can be used to optimize the calculation speed for some classes of integrals. We also provided examples of using of the presented package, including the applications in the geometry of quantum states. 
Calculation time of the package strongly depends on a degree of the integrand. For polynomials of small degree, the package is able to calculate the value of integral using the direct formula (3). For polynomials of large degree, the calculation time grows rapidly and the calculation is possible only if one of the special cases (optimizations) is used.

Nevertheless, the presented package can be very useful in the investigations involving circular unitary ensemble and the geometry of quantum states and quantum entanglement.

Acknowledgements. The work of Z. Puchała was partially supported by the Polish National Science Centre under research project N N514 513340, while the work of J. A. Miszczak was partially supported by the Polish National Science Centre under research project N N516 475440. Authors would like to thank K. Życzkowski and P. Gawron for motivation and interesting discussions

\section{REFERENCES}

[1] B. Collins and P. Śniady, "Integration with respect to the Haar measure on unitary, orthogonal and symplectic group", Commun. Math. Phys. 264, 773-795 (2006).

[2] N. Ullah and C. Porter. "Expectation value fluctuations in the unitary ensemble", Physical Review 132 (2), 948 (1963).

[3] D. Weingarten, "Asymptotic behavior of group integrals in the limit of infinite rank", Journal of Mathematical Physics 19, 999 (1978).

[4] Z. Puchała and J. A. Miszczak, "IntU package for Mathematica" (2011). Software available at http://zksi.iitis.pl/wiki/projects:intu.
[5] W. Fulton and J. Harris, Representation Theory: A First Course - Graduate Texts in Mathematics vol. 129, Springer Verlag (1991).

[6] G. James and A. Kerber, "The representation theory of the symmetric group", Encyclopaedia of Mathematics, vol .16 (1981).

[7] B. Sagan, The Symmetric Group: Representations, Combinatorial Algorithms, and Symmetric Functions, Springer Verlag (2001).

[8] D. Bernstein, "The computational complexity of rules for the character table of $S_{n}$ ", Journal of Symbolic Computation 37 (6), 727-748 (2004).

[9] F. Hiai and D. Petz, "The semicircle law, free random variables and entropy", Amer. Mathematical Society 77 (2006).

[10] C. Donati-Martin and A. Rouault, "Truncations of Haar unitary matrices, traces and bivariate Brownian bridge". Arxiv preprint, arXiv:1007.1366 (2010).

[11] Z. Puchała, J. A. Miszczak, P. Gawron, C. F. Dunk1, J. A. Holbrook, and K. Życzkowski, "Restricted numerical shadow and the geometry of quantum entanglement", Journal of Physics A: Mathematical and Theoretical 45 (41), 415309 (2012).

[12] J. Miszczak, "Generating and using truly random quantum states in Mathematica", Comput. Phys. Commun. 183 (1), 118-124 (2012).

[13] C. F. Dunkl, P. Gawron, J. A. Holbrook, Z. Puchała, and K. Życzkowski, "Numerical shadows: measures and densities on the numerical range", Linear Algebra Appl. 434, 2042-2080 (2011).

[14] C. F. Dunkl, P. Gawron, J. A. Holbrook, J. A. Miszczak, Z. Puchała, and K. Życzkowski, "Numerical shadow and geometry of quantum states", J. Phys. A: Math. Theor. 44 (33), 335301 (2011).

[15] M. Enríquez, Z. Puchała, and K. Życzkowski, "Minimal Rényi-Ingarden-Urbanik entropy of multipartite quantum states", Entropy 17 (7), 5063 (2015). 